\title{
Experimental Determination of Meteoric Line Densities and Attachment Rates
}

\author{
Laurence A. Manning \\ Contribution From the Radioscience Laboratory, Stanford University, Stanford, Calif. ${ }^{1}$
}

(Received March 19, 1964; revised May 25, 1964)

\begin{abstract}
A theory for the dependence of meteoric-echo heights and durations on meteor velocity and magnitude, and on the radio wavelength, is used to interpret experimental meteor data of a variety of types. It is shown that the data set forms a more consistent whole on the assumption of a three-body than of a two-body attachment process. No velocity dependence is found for the ratio of line density to luminosity. An attachment time constant of about $100 \mathrm{sec}$ is deduced for a reference height of $95 \mathrm{~km}$, and a zero-magnitude meteor is found to produce a maximum line density of about $100 \times 10^{14}$ electrons $/ \mathrm{m}$. Combination of the theoretical and experimental results makes possible calculation of the heights and durations of meteor echoes under a full range of conditions.
\end{abstract}

\section{Introduction}

In a previous paper [Manning, 1962] hereafter to be referred to as part $I$, the author developed a theory giving the theoretical heights and durations of radio echoes from large meteors. The theory assumed that trails are dispersed by attachment as well as diffusion. The scale height of the attachment process was made adjustable, as was the velocity dependence of the ratio of ionizing to luminous efficiency. In the present paper the theory will be compared with experimental results, and it will be shown that the unknown parameters of the theory can be determined. These parameters include the attachment time constant at reference height, the height dependence of the attachment time constant, the exponent of the velocity dependence of line density for fixed visual magnitude, the coefficient giving line density for reference visual magnitude, and a parameter relating height and maximum line density.

That attachment is an important process in meteor trails was shown by Davis, Greenhow, and Hall [1959a], and by Greenhow and Hall [1961]. They tested the effect of attachment by comparing theoretical curves, including attachment, with a variety of experimental plots. However, lacking a complete mathematical analysis of the echoing phenomena, they did not obtain all the data available from the experimental comparisons. Greenhow and Hall showed that a three-body attachment process was as good a fit to most of the data as was the two-body process of Davis, Greenhow, and Hall, but they favored the three-body process because it seemed to explain better the infrequency of enduring optical trains and of enduring radio echoes at low heights.

1 Jointly supported by the U.S. Army Signal Corps, the U.S. Air Force, the U.S. Navy (Office of Naval Research), and by the National Science Foundation, Grant NSF-GP948.
The author will show that a more thorough comparison of theory with the whole mass of experimental data is consistent only with a height dependence of attachment time constant equivalent to that for the three-body process. By solving simultaneously the relations fitting data of all types, best values will be found for the important parameters influencing echo decay. Insertion of the parameters in the theory of part I will make possible the prediction of echo behavior.

\section{Analysis of Duration Versus Visual Magnitude}

In part I the theory relating duration to line density was expressed in terms of a normalized duration variable $z$ and a normalized line-density variable $x$. For a given height-dependence of attachment time constant, a single curve was found relating $z$ to $x$, applicable for arbitrary wavelength, diffusion coefficient, meteor velocity, etc. By expressing the line density as a function of visual meteor magnitude, the theory can be compared with experimental measures of echo duration versus magnitude with velocity as a parameter. The values of the normalizing factors needed to fit the data to the theory will then determine several relations between the physical parameters of the trail. In making the analysis, we shall carry two sets of equations, one for two-body and the other for threebody attachment. Each type of experimental data will be compared with both sets of equations, so that a choice between the decay laws can finally be made by comparing the consistency of the sets of equations relating the derived parameters on the two assumptions. Two-body equation numbers will be written in the form (nn. 2), and the threebody equations as (nn. 3) where $\mathrm{nn}$ is the usual equation number. 
The normalized duration variables from part I while the normalized line-density variables are: are:

$$
z=2.2298 \frac{t_{H} D_{0}^{1 / 5} K^{3 / 5}}{A^{4 / 5} \lambda^{2 / 5} v^{(10+J) / 5}}
$$

and

$$
z=3.8058 \frac{t_{H} D_{0}^{1 / 3} K}{A^{2 / 3} \lambda^{2 / 3} v^{(10+J) / 3}}
$$

$$
x=\frac{1}{2.2298} \frac{Q_{m o} K^{6 / 5} \lambda^{6 / 5}}{A^{3 / 5} v^{2(10+J) / 5} D_{0}^{3 / 5}}
$$

and

$$
x=\frac{1}{7.4242} \frac{Q_{m_{0}} K^{3 / 2} \lambda}{A^{1 / 2} v^{(10+J) / 2} D_{0}^{1 / 2}}
$$

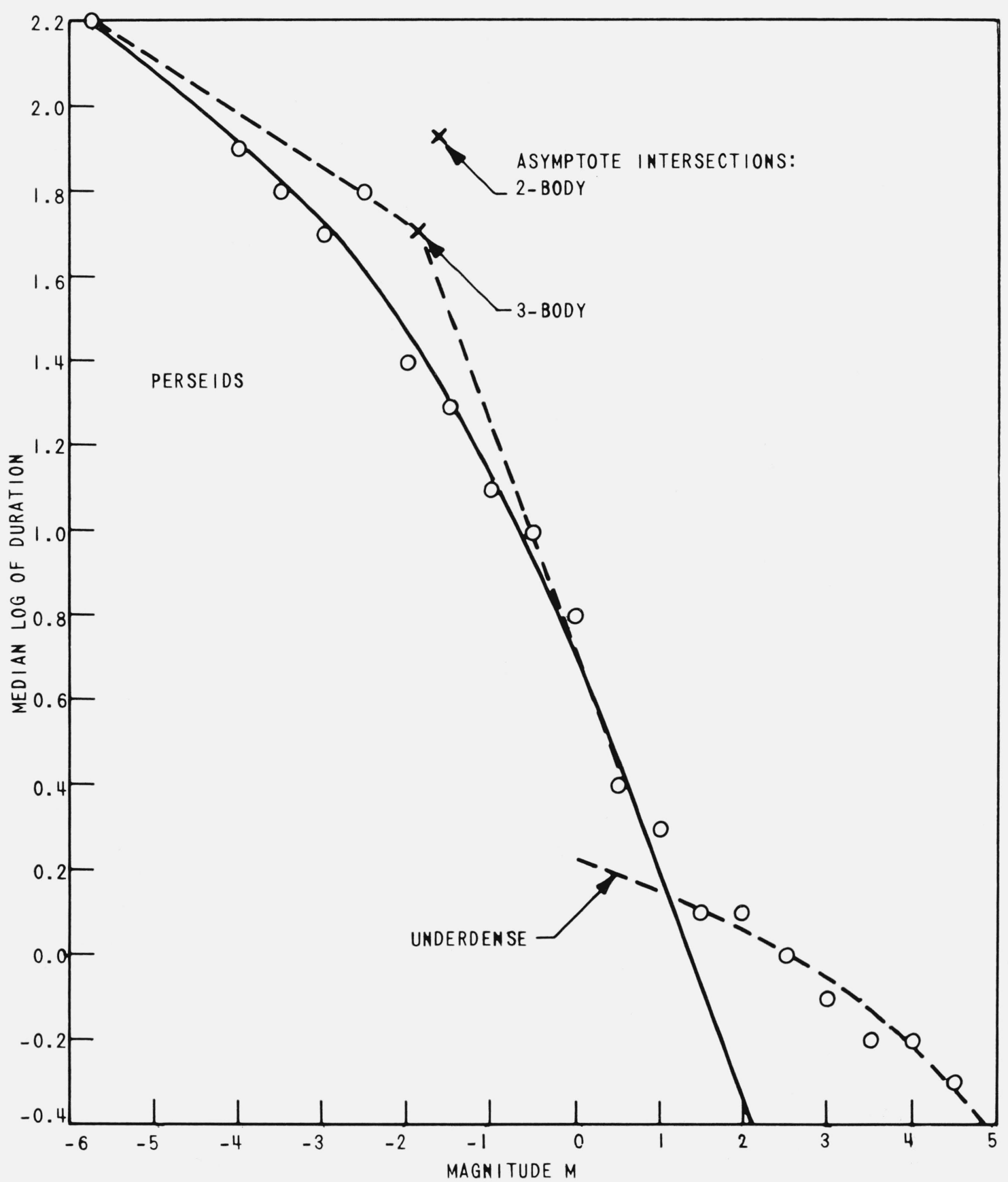

Figure 1. Median log (duration) versus magnitude from Millman and McKinley $[1956]$ for perseid meteors, V=60 km $/ \mathrm{s}$. The solid curve is a fitted theoretical three-body curve. Despite the difference in pcsition of the asymptote intersection, the two-body curve (not shown) fits almost as well. 
where $t_{H}$ is the echo duration, $D_{0}$ is the ambipolar diffusion coefficient at a reference height (to be taken as $95 \mathrm{~km}$ ), $K$ is a constant (defined by (11) of part I) in the equation relating the maximum line density to the height at which it occurs, $A$ is the attachment time constant at the reference height, $\lambda$ is the radio wavelength, and $v=\left(V / V_{0}\right)(\sec Z)^{2 /(10+J)}$, where $V$ is the meteor velocity, $V_{0}$ is a reference velocity, $Z$ is the meteoric zenith angle, and $J$ is the exponent of the velocity dependence of maximum normalized line density $Q_{m_{0}}=0.885 \times 10^{-14} Z$, with $Z$ the line density in electrons/meter.
As was shown in part I, the theoretical curves of $\log z$ versus $\log x$ are straight lines of different slope which are joined by a curved transition. To fit the experimental duration curves to theory, the normalized curves may be translated until they are superimposed on the experimental points with minimum error. The parameters of the fit may then be conveniently estimated from the duration and magnitude for which an extrapolation of the linear portions of the $\log z$ versus $\log x$ curves intersect. In part I it was shown that for two-body attachment the intersections occur at $x=1.3971, z=0.87772$, while for

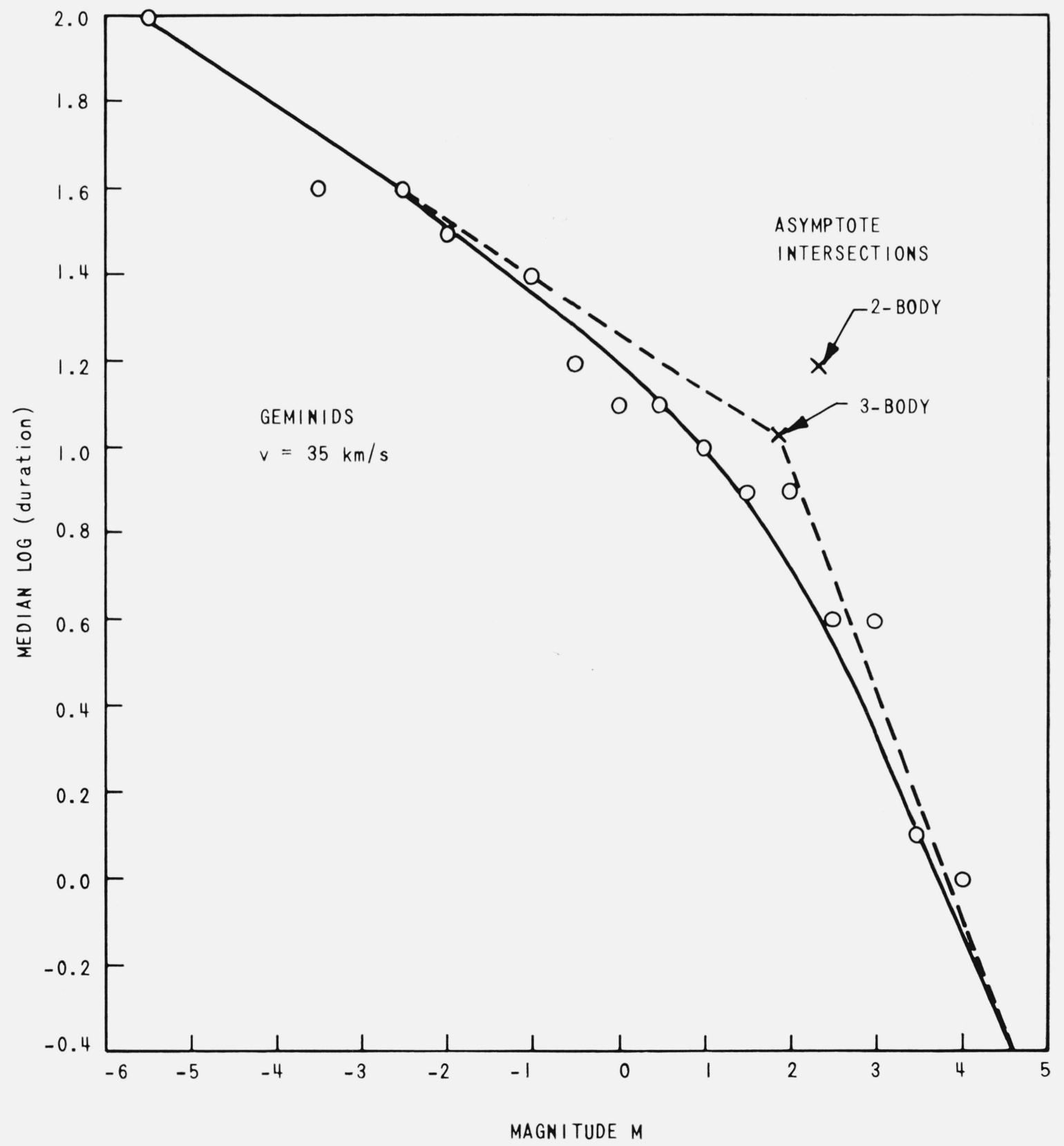

Figure 2. Median log (duration) versus magnitude from Millman and McKinley [1956] for geminid meteors, V=35 $\mathrm{km} / \mathrm{s}$. The smooth curve is fitted from the three-body theory. The two-body curve (not drawn) fits almost as well. 
three-body attachment the intersection occurs for $x=1.0783, z=0.62196$. These values of $x$ and $z$ may be substituted in (1) together with the duration and line-density parameters from the curve fitting. To relate the line density in (1) to the experimentally observed visual magnitude, use will be made of the equation

$$
Q_{m_{0}}=Q_{0}\left(V / V_{0}\right)^{J} 10^{-M / 2.5}
$$

when $Q_{m_{o}} \cong 10^{-14}$ times the maximum line density on the trail, $Q_{0}$ is a constant independent of velocity $V$ or magnitude $M$, but dependent on the choice of reference velocity $V_{0}$, and $J$ is a constant determining the velocity dependence of $Q_{m o}$; thus $J$ is the velocity exponent of the ratio of line-density to light output.

The best available experimental data on echo duration versus visual magnitude appears to be that of Millman and McKinley [1956]. They tabulate the logarithm of duration in steps of 0.2 versus absolute visual magnitude in steps of 0.5 for Perseid, Geminid, $\delta$ Aquarid, and nonshower meteors. The data on the Perseid (60 km/sec) and Geminid (35 $\mathrm{km} / \mathrm{sec}$ ) showers will be used in this study. The data on the Aquarids are based on a smaller number of meteors. For comparison with theory, the data of Millman and McKinley have been reduced by computing median values of duration for each magnitude interval. The resulting plots for the Perseids and Geminids are shown in figures 1 and 2 .

The smooth curve interpolated through the experimental points is a plot of the logarithm of normalized duration $z$ versus the logarithm of normalized line density $x$. The abscissa scale for $x$ was adjusted from $\log x$ proportional to $\log Q_{m o}$, to $\log x$ proportional to $-M / 2.5$ using (3). Separate theoretical curves of $\log z$ versus $\log x$ (fig. 3 of part $\mathrm{I}$ ) were fitted to figures 1 and 2 by sight for both twoand three-body attachment; the curves plotted are for the three-body law. No firm choice between the two- and three-body curves can be made on the basis of goodness of fit alone, although the three-body curves seem a bit better. The best-fitting curves can be described by the values of log-duration and visual magnitude at the intersection of the dashed extrapolated linear curves and are tabulated in table 1. Although the curves for the two-body case are

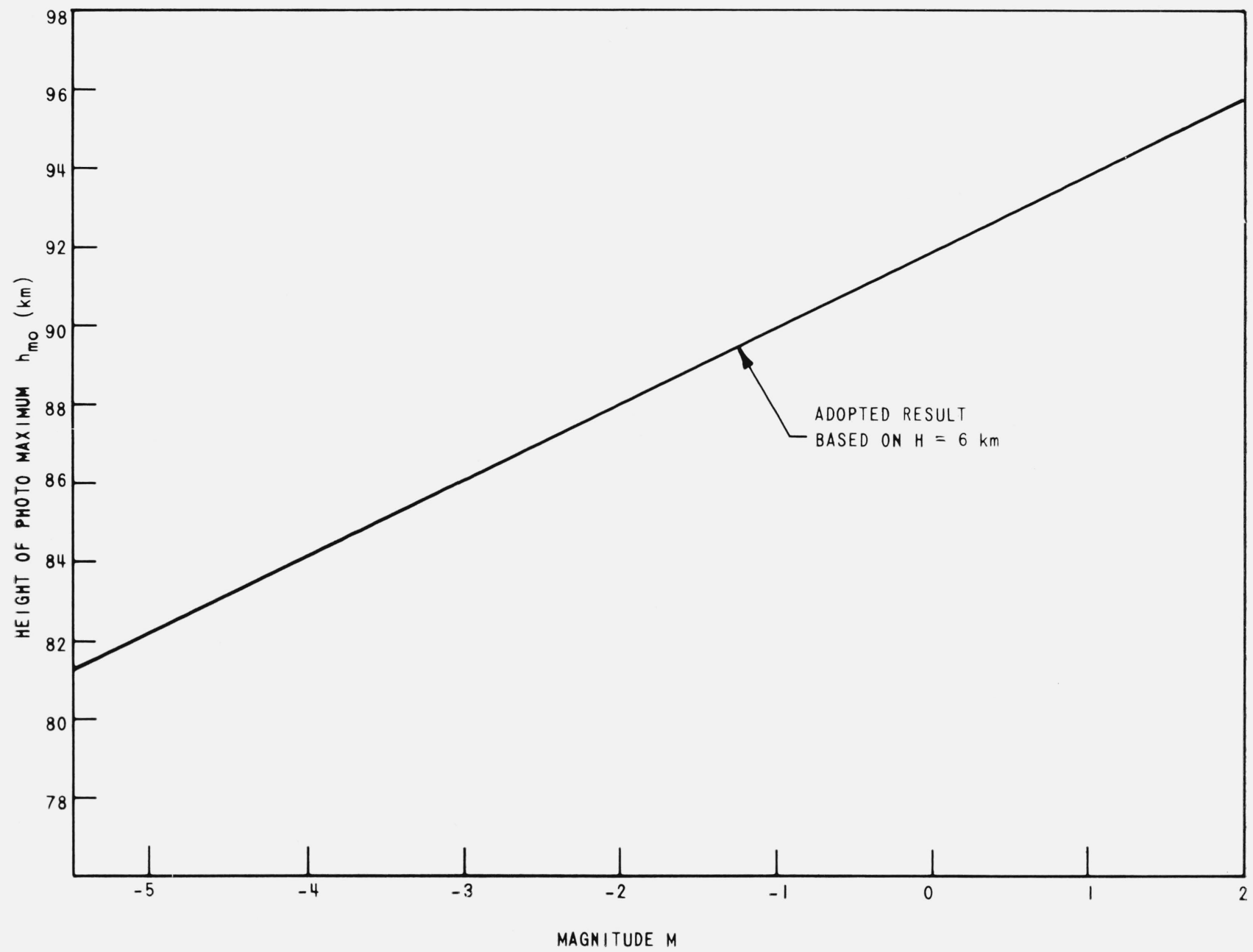

FIGURE 3. Height of maximum luminosity versus magnitude adopted as best fit to figure 5-11 of McKinley [1961] for fixed scale height. 
not plotted, the point of intersection is indicated by a small box. Note that in figure 1 the deviation of the points from the curve for negative log of duration is fitted to a theoretical curve based on under-dense behavior. Comparison with figure 2 shows that the transition from underdense to overdense duration behavior occurs at lower magnitudes for the Perseid meteors because of the smaller overdense duration for a given magnitude.

TABLE 1. Experimental results

\begin{tabular}{|c|c|c|}
\hline & $\begin{array}{l}\text { Two-body } \\
\text { attachment }\end{array}$ & $\begin{array}{l}\text { Three-body y } \\
\text { attachment }\end{array}$ \\
\hline Perseids & $\log t_{H 1}=1.93$ & $\log t_{H 1}=1.71$ \\
\hline Geminids. & $\begin{array}{c}M_{1}=-1.0 \\
\log t_{H 2}=1.19 \\
M_{2}=2.3\end{array}$ & $\begin{array}{c}M_{1}=-1.9 \\
\log t_{H 2}=1.02 \\
M_{2}=1.9\end{array}$ \\
\hline
\end{tabular}

Millman and McKinley's measurements were made with a radio wavelength of $\lambda=9.18 \mathrm{~m}$. At a reference height of $95 \mathrm{~km}$, we shall assume $D_{0}=$ $4 \mathrm{~m}^{2} / \mathrm{sec}$. Putting these constants in (1) and (2) together with the values of $z$ and $x$ previously given as applying to the intersection of the linear segments of the theoretical curves, we obtain in logarithmic form the pairs of equations.

$\frac{4}{5} \log A+\frac{(10+J)}{5} \log v=0.14+\frac{3}{5} \log K+\log t_{H}$

$\frac{3}{5} \log A+\frac{2(10+J)}{5} \log v=-0.396+\frac{6}{5} \log K+\log Q_{m o}$

and

$$
\frac{2}{3} \log A+\frac{(10+J)}{3} \log v=0.347+\log K+\log t_{H}
$$

$$
\frac{1}{2} \log A+\frac{(10+J)}{2} \log v=-0.241+\frac{3}{2} \log K+\log Q_{m 0} .
$$

Eliminating $\log v$ between (4) and (5), and eliminating $Q_{m_{o}}$ using (3),

$\log A=0.676+2 \log t_{H}-\log Q_{0}-J \log \left(V / V_{0}\right)+M / 2.5$

$\log A=1.523+3 \log t_{H}-2 \log Q_{o}$

$$
-2 J \log \left(V / V_{0}\right)+2 M / 2.5
$$

If we put the values of $t_{H}$ and $M$ for the Perseids and Geminids in (6), two pairs of equations result.
Subtracting,

$0=2\left(\log t_{H 1}-\log t_{H^{2}}\right)$

$$
+\left(M_{1}-M_{2}\right) / 2.5+J \log V_{2} / V_{1}
$$

$0=3\left(\log t_{H 1}-\log t_{H 2}\right)$

$$
+\left(M_{1}-M_{2}\right) / 1.25+2 J \log V_{2} / V_{1} .
$$

Inserting the durations and magnitudes from table 1 , together with the ratio $V_{2} / V_{1}$ of the Perseid-toGeminid velocity as $60 / 35$, (7) can be solved for the exponents $J$ of the velocity dependence of the ratio of line density to luminosity. It was shown in part I that the exponent of the ratio of ionizing to luminous efficiency is $J+1$.

$$
\begin{aligned}
& J=-0.34( \pm 0.5) \\
& J=-2.07( \pm 1.0) .
\end{aligned}
$$

The asterisk will be used in the equation numbers when the equations represent the results of a calculation leading to relations between the unknown parameters. The parentheses in (8) give the estimated probable errors of the results based on the propagation of the estimated scaling errors from figures 1 and 2 .

The values of $J$ from (8) may now be inserted in (4) and (5). Evaluating these equations for the Perseids and Geminids and subtracting, it is possible to solve for the ratio of $v_{60} / v_{35}$, the ratio of the values of

$$
v=\frac{V}{V_{0}(\cos Z)^{2 /(10+J)}}
$$

for 60 - and $35-\mathrm{km} / \mathrm{s}$ meteors; note, however, that the zenith angle $Z$ of the observations for Perseids and Geminids differs, so that $v_{60} / v_{35} \not \equiv V_{60} / V_{35}$. From subtraction of (4.2) for the two velocities,

$$
\frac{v_{60}}{v_{35}}=2.42
$$

and from (4.3)

$$
\frac{v_{60}}{v_{35}}=1.82 .
$$

Subtraction of (5) yields the same result. The significance of these results will be discussed after all the available results have been derived.

Another set of relations between unknown quantities may be obtained by eliminating $Q_{m o}$ from (5) using (3), and then eliminating $\log v$ between (4) and (5). The results are

$-\log A=-0.68+\log Q_{0}$

$$
-M / 2.5+J \log V / V_{0}-2 \log t_{H}
$$


$-\frac{1}{2} \log A=-0.763+\log Q_{0}$

$$
-M / 2.5+J \log V / V_{0}-(3 / 2) \log t_{H} .
$$

As a reference velocity we shall choose $V_{0}=35 \mathrm{~km} / \mathrm{sec}$. Thus $Q_{0}$ will be the maximum normalized line density of a zero-magnitude, $35-\mathrm{km} / \mathrm{sec}$ meteor. Then evaluating (11) for the values of $M, t_{H}$, and $V$ for Geminid meteors $\left(V=35 \mathrm{~km} / \mathrm{sec}\right.$, and $t_{H}, M$ from table 1), there results

$$
\begin{gathered}
\log A+\log Q_{0}=3.98 \\
0.5 \log A+\log Q_{0}=3.05 .
\end{gathered}
$$

These equations are independent of the value of $J$.

One additional independent result may be deduced from the duration-magnitude data. Putting $J$ from (8) into (4), and evaluating for Geminids of $V=35$, there results

$$
\begin{aligned}
\log K+2.22 & =\frac{4}{3} \log A+3.21 \log v_{35} \\
\log K+1.367 & =\frac{2}{3} \log A+2.65 \log v_{35} .
\end{aligned}
$$

The values of $v_{35}$ and $v_{60}$ can be roughly estimated independently of the duration-magnitude data. In response to a letter, Millman has kindly provided rough estimates of the values of zenith angle appropriate to the observations in Millman and McKinley [1956]. From his data, a rough mean value of zenith angle $Z$ would seem to be $Z=30^{\circ}$ for the Geminids, $Z=45^{\circ}$ for the Perseids. Fortunately $v$ is relatively insensitive to the value of $Z$. From (9), using $J$ from (8),

$$
\begin{aligned}
& v_{35}=1.03 \\
& v_{35}=1.04
\end{aligned}
$$

and

$$
\begin{aligned}
& v_{60}=1.85 \\
& v_{60}=1.86
\end{aligned}
$$

and

$$
\frac{v_{60}}{v_{35}}=1.80
$$

for two- or three-body theory. Comparison of (16) and (10) favors the three-body case.

Putting $V_{35}$ from (14) in (13) leads to the result

$$
\begin{aligned}
& \log K+2.16=\frac{4}{3} \log A \\
& \log K+1.336=\frac{2}{3} \log A .
\end{aligned}
$$

The starred equations may be looked on as part of a set relating the attachment and trail-formation parameters. Other relations between these parameters will be derived in the following sections from other forms of experimental data, and then the most consistent solutions to the resultant over-determined sets of equations will be found.

In evaluating (2) the diffusion coefficient $D_{0}$ at the reference height $95 \mathrm{~km}$ has been assumed to be
$4 \mathrm{~m}^{2} / \mathrm{sec}$. This value is consistent with both diffusion theory and experimental values deduced from underdense-echo amplitude decay versus time. However, using the data of figure 1 the chosen value of $D_{0}$ may be verified while elucidating the properties of the duration-magnitude plots. For underdense echoes, the echo amplitude is proportional to $Q_{m o} \exp (-t / T)$ where $T$ is the exponential-decay time constant, $\lambda^{2} /\left(16 \pi^{2} D\right)$. Thus using (3), echo amplitude in the underdense region will be proportional to $\exp (-0.92 M-t / T)$. The duration will be determined by the moment when the amplitude falls below a threshold set by the system sensitivity. Hence, if $t$ is set equal to the underdense duration, it follows that $0.92 M+t_{H} / T=C$, a system-sensitivity constant. If duration is plotted versus $M$ on a linear scale, the decay time constant $T$ can be found. Plotting the durations less than 1.5 sec versus $M$ from figure 1 vields $C=5.8, T=0.29$ sec. From $T$, with $\lambda=9.18$, the diffusion coefficient is $1.8 \mathrm{~m}^{2} / \mathrm{sec}$. From McKinley [1961, fig. 5-11], for $M=2.5$ the height is just above $90 \mathrm{~km}$. Correcting $D=1.8$ $\mathrm{m}^{2} / \mathrm{sec}$ at $90 \mathrm{~km}$ to the reference height of $95 \mathrm{~km}$ using a scale height of $6 \mathrm{~km}$ gives $D_{0}=4.1$, close to the value adopted. In figure 1 the underdense durations as fitted above are plotted as the dashed line. It is interesting to note that the duration and magnitude at which the transition from underdense to overdense echo behavior occurs is made clearly evident by this procedure. The position of the transition depends on meteor velocity and is not the same for Perseids and Geminids.

\section{Height of Maximum Light Production}

The photographic evidence on the height of maximum light production may be used to derive another relation between trail parameters. From (11) of part I,

$$
\frac{K Q_{m o}^{1 / 3}}{v^{(10+J) / 3}}=\exp \left[\left(95-h_{m o}\right) / H\right]
$$

where $Q_{m o}$ is the maximum value of normalized line density on the trail, $h_{m_{o}}$ is the height of maximum line density (and light production), $v$ is defined by (9), and, as before, $J$ is the velocity exponent of the ratio of line density to luminosity, $H$ is the atmospheric scale height, and the parameter $K$ may be considered defined by the equation. Its value depends in a complicated way on the meteoric density, ionization potential, heat of ablation, heattransfer coefficient, shape factor, etc.

Eliminating $Q_{m_{o}}$ using (3), and setting $\left(V / V_{0}\right) \cong v$, gives

$$
\log \left(K Q_{0}^{1 / 3} / v^{10 / 3}\right)-M / 7.5=0.436 \frac{\left(95-h_{m o}\right)}{H} .
$$

If $v$ does not change with magnitude $M$, and $H$ is independent of height, (19) describes a linear relation between $h_{m o}$ and $M$. In figure 3 a straight line has been fitted to the height data of Whipple, Jacchia, and Hawkins, and Southworth as summa- 
rized by Millman and McKinley [1956, fig. 5-11]. The parameters of the fit are $H=6 \mathrm{~km}, h_{m_{o}}=92 \mathrm{~km}$ at $M=0$. Equation (19) then becomes

$$
\log K+\frac{1}{3} \log Q_{0}=0.218+\frac{10}{3} \log \bar{v}
$$

Note that the experimental observations relate to meteors with a distribution in velocity. Thus the symbol $\bar{v}$ refers to a mean value of $v$.

\section{Height of Enduring Radio Echoes}

The experimental results on the dependence of the height of enduring radio echoes versus visual magnitude are summarized by Millman and McKinley [1956, fig. 5-11]. In the presence of attachment and diffusion, the final echoing height $h_{m}$ differs from the height of maximum initial line density $h_{m o}$; the loss of electrons by attachment is greater at low heights, but the dispersal due to diffusion is greater at high altitudes. Experimentally, the final echoing height is nearly independent of visual magnitude, despite the marked increase in $h_{m o}$ with magnitude shown in figure 3 . Using the notation $u=\exp$ $\left[-\left(h-h_{m_{0}}\right) / H\right]$ defined by $(6)$ of part I, the difference in height between the final echoing point and the point of maximum initial line density may be described by $u_{m}=\exp \left[-\left(h_{m}-h_{m o}\right) / H\right]$. In figure 4 the quantity $-M / 2.5$ is plotted versus $\log u_{m}$, based on McKinley's plot.

Analysis of the data in figure 4 starts with (2). Eliminating $Q_{m}$ in favor of $Q_{0}$ and $M$ using (3), letting $v \cong V / V_{0}$, and taking logarithms,

$$
\begin{aligned}
& \log x=-\frac{M}{2.5}+\log \frac{Q_{0}(K \lambda)^{6 / 5}}{2.2298^{3}\left(A D_{0}\right)^{3 / 5} v^{(4-3 J / 5)}} \\
& \log x=-\frac{M}{2.5}+\log \frac{Q_{0} K^{3 / 2} \lambda}{7.4242\left(A D_{0}\right)^{1 / 2} v^{(5-J / 2)}} .
\end{aligned}
$$

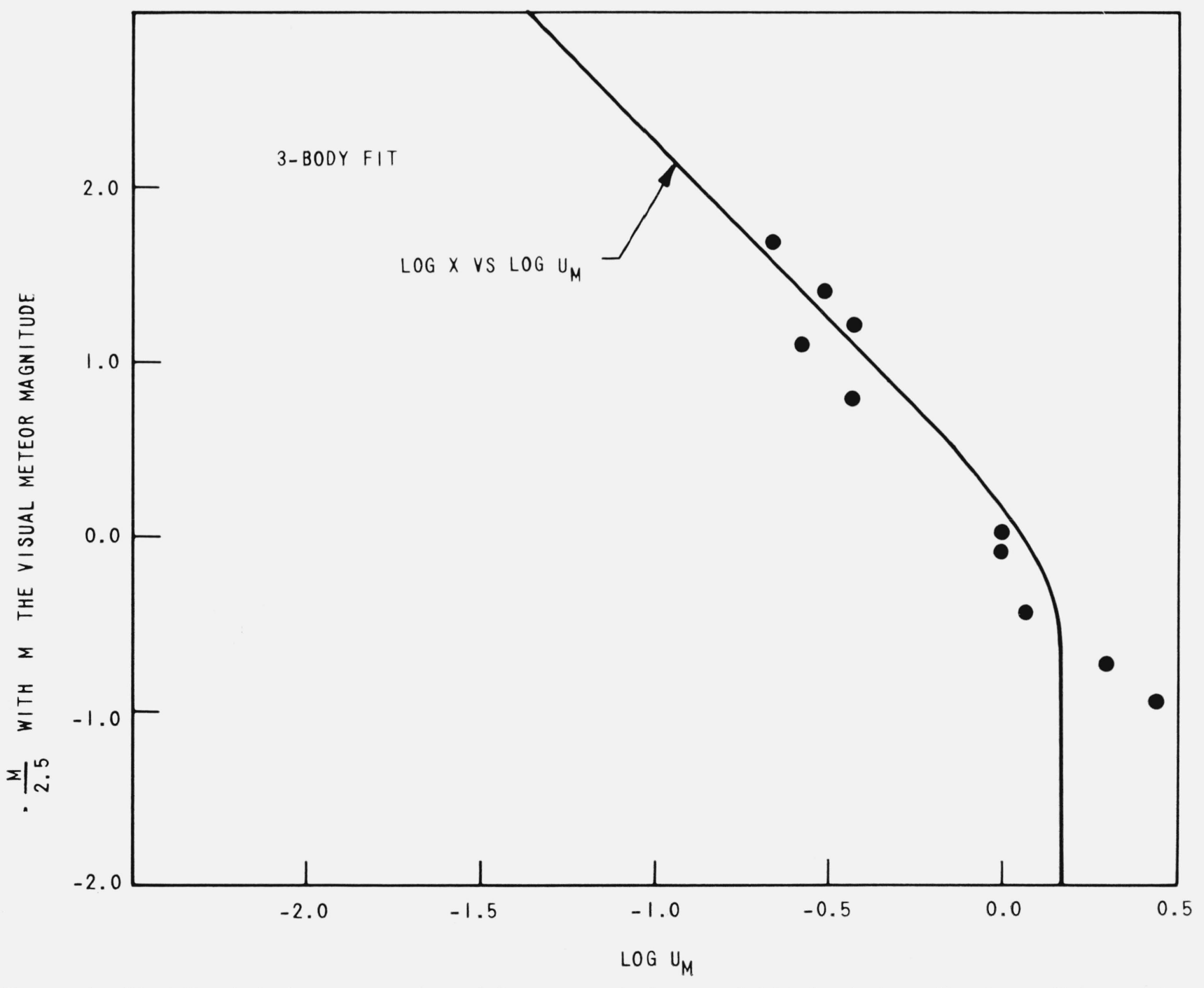

Figure 4. Fit of three-body theory to experimental data on magnitude versus height of enduring radio echoes. Points are from Millman and McKinley [1956]. 
Notice that $\log x$ and $-M / 2.5$ vary together linearly. Hence, the theoretical shape of the plot of $-M / 2.5$ versus $\log u_{m}$, in figure 4 will be the same as that of a plot of $\log x$ versus $\log u_{m}$, except for translation of the ordinate by an amount related to the bracketed quantity in (21). The theoretical variation of $\log x$ versus log $u_{m}$ is shown in figure 5 of part I, and the best-fitting curve for three-body attachment is included in figure 4 of the present part. No difference in goodness of fit is discernible between the two- and three-body cases. The best-fitting translation, for the logarithms of the bracketed quantities in (21), yields the values 0.35 for the two-body case, and 0.0 for the three-body case. Using $D_{0}=4 \mathrm{~m}^{2} / \mathrm{sec}$, and $\lambda=9.18 \mathrm{~m}$, the result is

$\log Q_{0}+\frac{6}{5} \log K-\frac{3}{5} \log A=0.60+\left(4-\frac{3 J}{5}\right) \log \bar{v}$

$\log Q_{0}+\frac{3}{2} \log K-\frac{1}{2} \log A=0.21+\left(5-\frac{J}{2}\right) \log \bar{v}$.

The velocity ratio $v$ has been barred because the data were taken for meteors of distributed velocity. An estimate of the mean velocity in figure 5-11 of

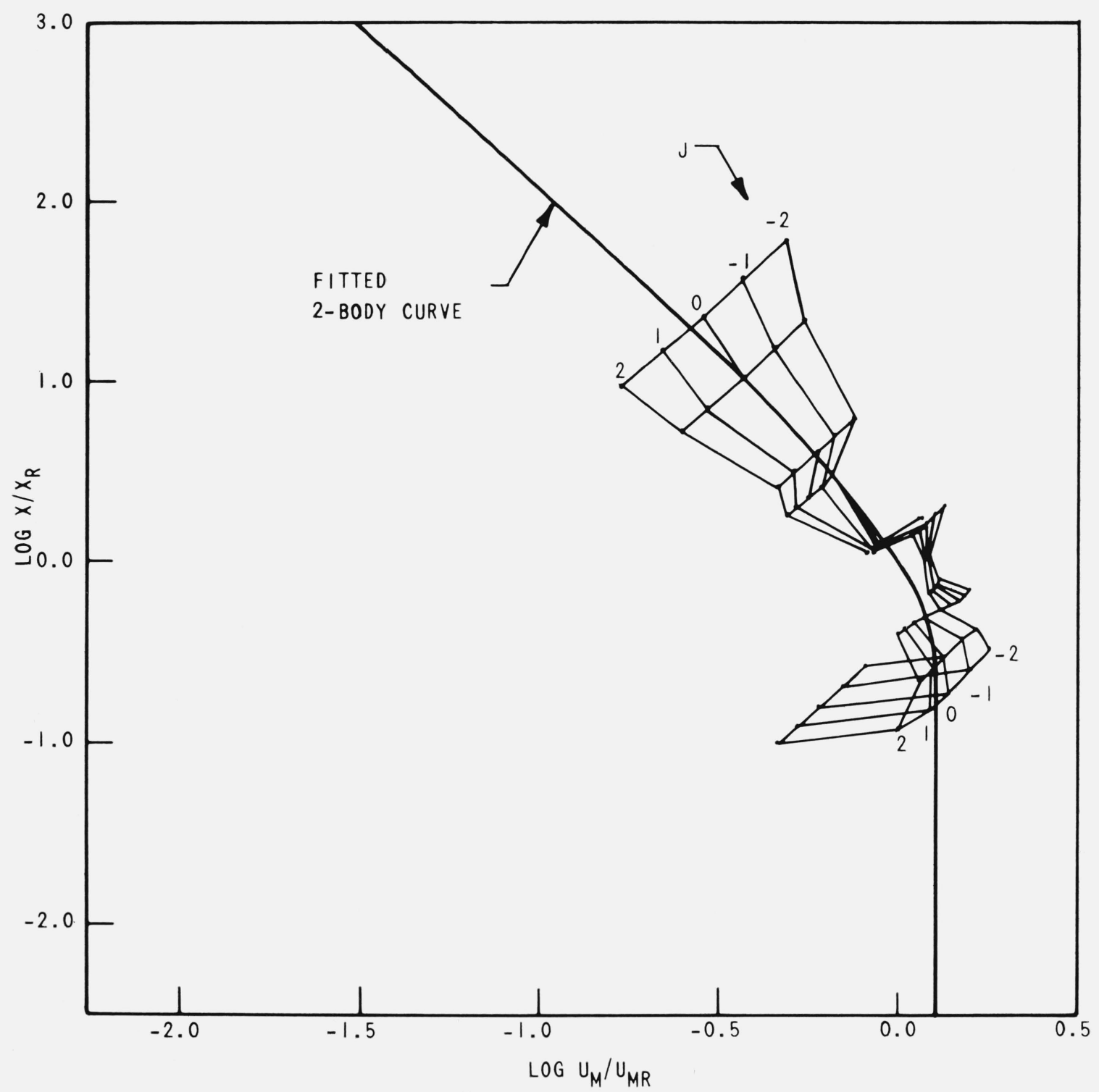

FIGURE 5. Fit to the theory of processed two-body velocity-versus-height data. 
McKinley can be found from his figure 5-9; the heights in figure 5-11 for a given magnitude correspond to a velocity $V$ of about $43 \mathrm{~km} / \mathrm{sec}$. Using $V_{0}=35 \mathrm{~km} / \mathrm{sec}$, an estimate for $\bar{v}$ in $\left(22^{*}\right)$ and $\left(20^{*}\right)$ is then

$$
\bar{v}=1.23 .
$$

This value may be used as a rough check to the values of $v$ to be deduced later by simultaneous solution of the starred equations.

\section{Height Versus Velocity Data}

As meteor velocity increases, there is a corresponding increase in both the height of maximum initial ionization production as determined from the height of maximum light production, and the height of enduring radio echoes. Analysis of these data will yield additional relations between the trail parameters, including an independent estimate of $J$.

The basic height equation, obtained from (18) by multiplying both sides by $u_{m}=\exp \left[-\left(h_{m}-h_{m_{o}}\right) / H\right]$, is

$$
\frac{K Q_{m o}^{1 / 3} u_{m}}{v^{(10+J) / 3}}=\exp \left[\left(95-h_{m}\right) / H\right]
$$

with $h_{m}$ the final echoing height.

Since the radar sensitivity fixes the detectable line density parameter $Q_{m o}$, it is the same for all

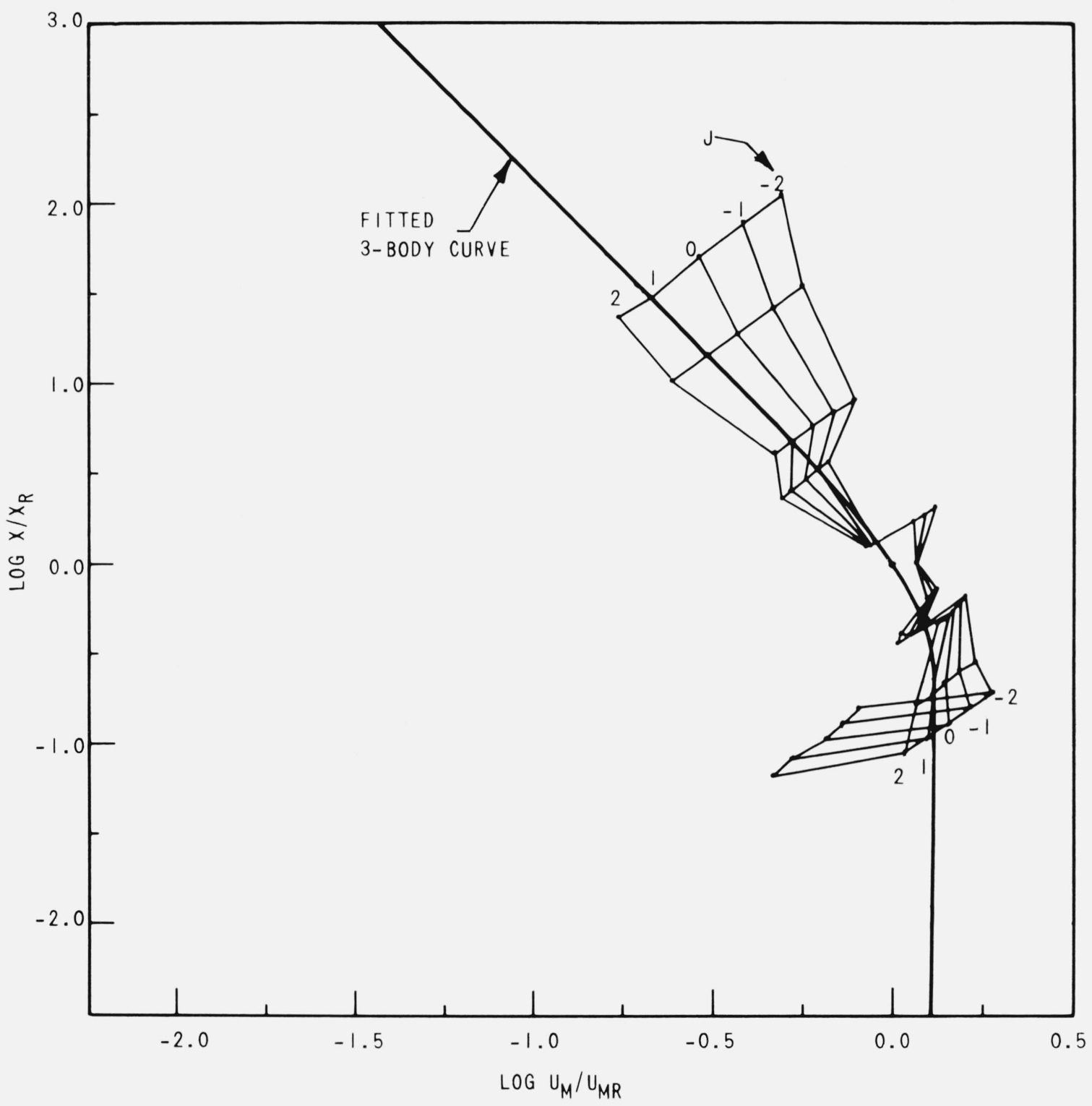

FiguRE 6. Fit to the theory of processed three-body velocity-versus-height data. 
velocities, and with respect to a reference height and velocity,

$$
\frac{u_{m}}{u_{m \boldsymbol{R}}}=\left(\frac{v}{v_{R}}\right)^{(10+J) / 3} \exp \left[\left(h_{m R}-h_{m}\right) / H\right]
$$

where $u_{m R}=\exp \left[-\left(h_{m R}-h_{m o R}\right) / H\right]$ is a measure of the difference of the enduring radio and visual heights at the reference velocity. With the exception of $J$, the quantities on the right-hand side of (25) are known, since figure 5-10 of McKinley [1961] gives radio ending heights versus velocity. The ratio $u_{m} / u_{m R}$ on the left-hand side of (25) is a function of $x$ determined by the theory of part I. But from (2), upon eliminating $Q_{m_{o}}$ by (3),

$$
\begin{aligned}
& \frac{x}{x_{R}}=\left(\frac{v}{v_{R}}\right)^{(3 J / 5)-4} \\
& \frac{x}{x_{R}}=\left(\frac{v}{v_{R}}\right)^{(J / 2)-5} .
\end{aligned}
$$

Then $u_{m} / u_{m R}$ versus $x / x_{R}$ from (25) can be solved simultaneously with the theoretical relation between the same variables for a variety of assumed values of $J$. Each experimental point of height $h_{m}$ versus velocity $V$ determines a value of $\left(v / v_{R}\right)$ and of $\left(h_{m R}-h_{m}\right)$ once the reference height and velocity are chosen; the values used are $40 \mathrm{~km} / \mathrm{sec}$ and $94 \mathrm{~km}$. For each assumed value of $J$, the experimental points determine a value $x / x_{R}$ from (26), and a value of $\left(u_{m} / u_{m R}\right)$ from (25). The values of log $\left(x / x_{R}\right)$ versus log $\left(u_{m} / u_{m R}\right)$, for $-2 \leq J \leq 2$ were calculated by digital computer and are plotted in figures 5 and 6 for two- and three-body attachment. The theoretical curve of $\log x$ versus $\log u_{m}$ (fig. 5 of part I) was then superimposed on the plots of figures 5 and 6 and translated in both coordinate directions to obtain the best fit. Because in figures 5 and $6 x=x_{R}$, and $u_{m}=u_{m R}$ is the origin, and because the required translations of the theoretical $\log x$ versus $\log u_{m}$ curves are $\log x_{R}$ and $\log u_{m R}$, the theoretical $\log x$ versus $\log u_{m}$ curve must be translated so that it always passes through the origin in the $\log \left(x / x_{R}\right)$ versus $\log \left(u_{m} / u_{m R}\right)$ plots. The experimental curve of log $\left(x / x_{R}\right)$ versus $\log \left(u_{m} / u_{m R}\right)$ that can be best fitted to a translated theoretical curve will correspond to the best value of $J$. The translation $\log x_{R}$ (or the related translation log $u_{m R}$ ) can be used to determine another relation between the trail parameters.

For two-body attachment, the best fit to figure 5 occurs if $J$ equals about 0 or 0.5 , with 0.5 more likely, and $\log x_{R}=0.3, \log u_{m R}=0.075$. For threebody attachment, the best fit occurs with $J=1$, and $\log x_{R}=0.0, \log u_{m R}=0.05$. The determinations of $J$ made in this way,

$$
\begin{aligned}
& J=0.5 \\
& J=1.0
\end{aligned}
$$

are based on data of an entirely different type than are the determinations of (8). Comparison $(J=$
$-0.34,-2.07)$ with the present values suggests adoption of $J=0$ as consistent with the accuracies of both sets of data. However, in interpreting $x_{R}$ and $u_{m R}$ from the velocity measure, the values of $J$ in $(27)$ will be retained to insure the independence of the resulting measures.

By definition, $u_{m R}=\exp \left[\left(h_{m o R}-h_{m R}\right) / H\right]$. Thus the scaled values of $u_{m R}$ determine the difference between the maximum visual height and the radio enduring height. Assuming the scale height $H=6$ $\mathrm{km}, h_{m o R}-h_{m R}$ has the value $0.42 \mathrm{~km}$ for two-body attachment, and $0.69 \mathrm{~km}$ for three-body. Referring now to McKinley [1961, fig. 5-9], it is found that the height of maximum light production is between 93 and $94 \mathrm{~km}$ for meteors in the magnitude class from zero to two. Weighting the mean magnitude in the class interval by the relative frequency of meteors versus magnitude gives a mean magnitude $M=1.4$ corresponding to the radio height-velocity data analyzed.

Now the scaled values of $x_{R}$ may be interpreted using (2) for defining $x$, and (3) to eliminate $Q_{m o}$. The value of $v=(40 / 35)(\sec Z)^{2 /(10+J)}$ will be taken to be $1.2, M=1.4$. For two-body attachment with $\log x_{R}=0.3$, and for three-body with $\log x_{R}=0.0$, the resulting equations are

$$
\begin{aligned}
& \log Q_{0}+\frac{6}{5} \log K=\frac{3}{5} \log A+1.41 \\
& \log Q_{0}+\frac{3}{2} \log K=\frac{1}{2} \log A+1.14 .
\end{aligned}
$$

The constant $Q_{0}$ is defined for a reference velocity of $35 \mathrm{~km} / \mathrm{sec}$.

\section{Wavelength Dependence of Duration}

Meteor-echo durations are greater at larger wavelengths than at shorter wavelengths. However, the difference in the logarithm of duration is less for long- than for short-duration meteors. The theoretical variation of the exponent $n$ of the wavelength dependence decreases with $\log z$, as shown in figure 6 of part I. The exponent theoretically equals two for short-duration echoes, and for long-duration echoes $n$ approaches $2 / 3$ on the two-body theory, 1 on the three-body theory.

There are two principal sources of experimental data on the wavelength exponent. Greenhow [1952] determined the wavelength exponent $n$ defined by the expression $t_{H_{1}} / t_{H_{2}}=\left(\lambda_{1} / \lambda_{2}\right)^{n}$ at $\lambda=4$ and $8 \mathrm{~m}$, and plotted a histogram of $n$ versus the 4-meter duration. His histogram shows $n=2.25$ at duration approaching zero, and $n=0.85$ for durations of 32 to 64 sec. Thus Greenhow's results for high durations are intermediate between the two- and three-body limits, and do not directly discriminate between the two cases. Greenhow obtained $n=1.5$ for $t_{H} \cong 4$ sec. McKinley [1953, fig. 4] plots the logarithm of the ratio of echo duration on 9.22- and $5.35-\mathrm{m}$ wavelength versus the logarithm of the $5.35-\mathrm{m}$ duration. His amplitude-corrected curve has $n=2$ at about $t_{H}=0.5 \mathrm{sec}$, and drops to $n \cong 1.1$ at $t_{H} \cong 100 \mathrm{sec}$, thus fitting the three-body asymptote 
better than the two-body asymptote. For $\lambda=$ $5.35 \mathrm{~m}$, McKinley gets $n=1.5$ at an estimated duration of $8 \mathrm{sec}$, which corrects to $6.5 \mathrm{sec}$, in fair agreement with Greenhow's 4 sec at $\lambda=4 \mathrm{~m}$. The geometric mean of the two observations is 5.1 sec at $4 \mathrm{~m}$. From an expanded plot of figure 6 of part I (obtained using (41) and (42), and interpolation of table 1 ), $n=1.5$ corresponds to log $z \cong-0.575, z \simeq 0.266$ for both two- and three-body attachment. Putting this $z$ in (1) together with $\lambda=4.0, D_{0}=4.0, t_{H}=5.1$, and taking logarithms,

$$
\begin{aligned}
& \log K+1.086=\frac{10+J}{3} \log \bar{v}+\frac{4}{3} \log A \\
& \log K+1.66=\frac{10+J}{3} \log \bar{v}+\frac{2}{3} \log A .
\end{aligned}
$$

These equations refer to observations made for a mean normalized velocity $\bar{v}$.

\section{Single-Meteor Data}

The experimental results that have been considered so far are of a statistical nature. However, given sufficient information concerning a single meteor, it is possible to obtain an independent estimate of trail properties. Davis, Greenhow, and Hall [1959b], by combined radar and photographic observation, have obtained such information for one meteor. From their text and figures the following parameters can be found: $h_{\mathrm{mo}}=81 \mathrm{~km}$ (they scaled $79.2), h_{m}=94.9 \mathrm{~km}, t_{H}=46 \mathrm{sec}, M=-3.5$ (smoothing their curve), $V=35.2 \mathrm{~km} / \mathrm{sec}, \quad \lambda=8 \mathrm{~m}, \quad Z \simeq 20^{\circ}$ (from their figure).

From the height data, and using a scale height $H$ of $6 \mathrm{~km}, u_{m}=\exp \left[-\left(h_{m}-h_{m o}\right) / H\right]=\exp (-2.33)=$ -1.01. From figure 5 of part I it follows that log $x=2.5$ or 2.2 for two- or three-body attachment, and from figure 3 of part I (or table 1) $\log z=0.47$ or 0.50 . The value of $v$ is $\left(V / V_{0}\right)(\sec Z)^{2 /(10+J)}$; with $V=35.2, V_{0}=35, Z=20^{\circ}$, and $J=0, v=1.012$. From (1), substitution of the preceding values yields

$$
\begin{aligned}
& \log K+1.71=\frac{4}{3} \log A \\
& \log K+1.33=\frac{2}{3} \log A
\end{aligned}
$$

without use of the magnitude $M$. Substitution in (2) with $Q_{m o}$ from (3) yields

$$
\begin{aligned}
& \log Q_{0}+\frac{6}{5} \log K=\frac{3}{5} \log A+1.442 \\
& \log Q_{0}+\frac{3}{2} \log K=\frac{1}{2} \log A+1.392
\end{aligned}
$$

assuming $M=-3.5$ (as scaled from the brightness curve of Davis, Greenhow, and Hall [1959a]). However, in their text, p. 123, Davis et al. refer to the meteor as of magnitude -2 . It may be noted that (31.2) is almost identical to (28.2); (31.3) would be identical to (28.3) based on the height versus velocity data if $M=-2.8$, the average of -2 and -3.5 . Because of the uncertainty in the exact value of $M$, (31) will not be used as part of the set of experimentally derived relations, and it will be assumed that (28) contain results consistent with the single-meteor data as well as with the height-versus-velocity data.

\section{Summary of Results}

By comparing a number of different types of experiments a large number of equations have been derived relating the parameters of the trail process. In order to investigate the consistency of the equations, and to order their solution, it will be helpful to list the results that have been obtained in the order in which they will be used.

From duration versus magnitude

$$
\begin{aligned}
& J=-0.34( \pm 0.5) \\
& J=-2.07( \pm 1.0) .
\end{aligned}
$$

From radio height versus velocity

$$
\begin{aligned}
& J=0.5 \\
& J=1.0 .
\end{aligned}
$$

From duration versus magnitude

$$
\begin{aligned}
& \log K+2.16=(4 / 3) \log A \\
& \log K+1.33=(2 / 3) \log A .
\end{aligned}
$$

From the single-meteor data (independent of magnitude)

$$
\begin{aligned}
& \log K+1.71=(4 / 3) \log A \\
& \log K+1.33=(2 / 3) \log A .
\end{aligned}
$$

From single-meteor data (if $M=-3.5$ )

$$
\begin{aligned}
& \log Q_{0}+(6 / 5) \log K=(3 / 5) \log A+1.44 \\
& \log Q_{0}+(3 / 2) \log K=(1 / 2) \log A+1.39
\end{aligned}
$$

From radio height versus velocity

$$
\begin{aligned}
& \log Q_{0}+(6 / 5) \log K=(3 / 5) \log A+1.41 \\
& \log Q_{0}+(3 / 2) \log K=(1 / 2) \log A+1.14
\end{aligned}
$$

From duration versus magnitude

$$
\begin{gathered}
\log A+\log Q_{0}=3.98 \\
(1 / 2) \log A+\log Q_{0}=3.05 .
\end{gathered}
$$

From duration versus wa velength

$$
\begin{aligned}
& \log K+1.09=\frac{10+J}{3} \log \bar{v}+\frac{4}{3} \log A \\
& \log K+1.66=\frac{10+J}{3} \log \bar{v}+\frac{2}{3} \log A .
\end{aligned}
$$


From height versus velocity and magnitude

$$
\bar{v}=1.23 \text {. }
$$

From radio end height versus magnitude

$\log Q_{0}+(6 / 5) \log K-(3 / 5) \log A=0.60$

$$
+(4-3 J / 5) \log \bar{v}
$$

$\log Q_{0}+(3 / 2) \log K-(1 / 2) \log A=0.21$

$$
+(5-\mathrm{J} / 2) \log \bar{v} \text {. }
$$

From height of maximum light production versus magnitude

$$
\log K+(1 / 3) \log Q_{0}=0.22+(10 / 3) \log \bar{v} .
$$

From duration versus magnitude

$$
\begin{aligned}
& v_{60} / v_{35}=2.42 \\
& v_{60} / v_{35}=1.82 .
\end{aligned}
$$

Based on radiants in the letter from Millman

$$
\begin{gathered}
v_{60} / v_{35}=1.8 \\
v_{35}=1.03 \quad v_{60}=1.85 \quad\left(14.2^{*}\right) \\
v_{35}=1.04 \quad v_{60}=1.86 \quad\left(14.3^{*}\right),
\end{gathered}
$$

\section{Treatment of Three-Body Equations}

In this section the three-body relationships summarized in the preceding section will be examined for consistency, leaving the two-body equations to be treated in the following section. A comparison of the degree of consistency of the two sets of relations will be used to deduce whether the two-body or threebody attachment law is in better agreement with experiment. In addition, the most probable values of the constants $J, K, A$, and $Q_{\mathrm{o}}$ will be sought.

Two independent determinations have been made of $J$, the exponent of the velocity dependence of the ratio of line density to luminosity. From $\left(8.3^{*}\right)$ of the duration-versus-magnitude data, $J=-2.07$, with an uncertainty of \pm 1.0 attributed to the uncertainty of curve fitting to the given data. An uncertainty of twice as much is not inconsistent with the nature of the data. On the other hand, the value $J=1.0$ was found from $\left(27.3^{*}\right)$ of the radio-height versus velocity data. Negative values of $J$ could not reasonably be fitted to the height-velocity data, although $J=0$ might, and $J=2$ could fit rather well. It appears that $J=0$ is the result most consistent with the two sources of data. This value will be used where $J$ appears in the subsequent analysis, but the remaining equations are quite insensitive to the value of $J$ used.

Notice next that $\left(17.3^{*}\right)$, based on duration versus magnitude, is identical to $\left(30.3^{*}\right)$ based on the single- meteor data. Hence, (17.3) will be dropped from the set. Equation (31.3) of the single-meteor data is the same as $\left(28.3^{*}\right)$ of the radio height versus velocity data except for the constant term. As previously mentioned, a not unreasonable adjustment of the rather uncertain magnitude used in deriving (31.3) would make it identical with $\left(28.3^{*}\right)$. Hence (31.3) will not be used.

Examination of (12.3), (30,3), and (28.3) shows that (12.3) plus $3 / 2$ times (30.3) should equal (28.3). That is, $\left(0.5 \log A+\log Q_{0}-3.05\right)+(3 / 2)\left(\log K-\frac{2}{3}\right.$ $\log A+1.33) \equiv\left(\log Q_{0}-0.5 \log A+1.5 \log K-1.05\right)$ should equal $\left(\log Q_{0}-0.5 \log A+1.5 \log K-1.14\right)$. Thus $\left(23.3^{*}\right)$ differs from the equation that would be deduced from (12.3) and (30.3) only in that the constant 1.14 is 0.09 greater than calculated. It therefore appears reasonable to drop $\left(28.3^{*}\right)$ from the set, as being a dependent and reasonably consistent equation.

Next notice that (29.3) less (30.3) yields

$$
0.33=\frac{10+J}{3} \log v
$$

and taking $J=0, \bar{v}=1.26$. This is in excellent agreement with the independent estimate $\bar{v}=1.23$ of $\left(23^{*}\right)$. Thus (29.3) and (30.3) are consistent, and it will be convenient to eliminate (29.3) from the set. Now putting $\bar{v}=1.26$ and $J=0$ in (22.3) yields

$$
-\frac{1}{2} \log A+\log Q_{0}+\frac{3}{2} \log K=0.71
$$

in place of (22.3). Subtracting (32.3) from $\left(12.3^{*}\right)$ yields

$$
\log A-\frac{3}{2} \log K=2.34 \text {. }
$$

Comparing this equation with $3 / 2$ times (30.3), which is $\log A-1.5 \log K=2.0$, an approximate agreement is evident and (32.3) will be dropped. The remaining equations in $A, K$, and $Q_{0}$ are (12.3), (30.3), and (20). Inserting $\bar{v}=1.26$ in $\left(20^{*}\right)$, a set of three equations in three unknowns results:

$$
\begin{gathered}
0.5 \log A+\log Q_{0}=3.05 \\
-\log K+0.67 \log A=1.33 \\
\log K+0.33 \log Q_{0}=0.55 .
\end{gathered}
$$

Solution of this set yields $\log A=1.72, A=52.5 \mathrm{sec}$; $\log Q_{0}=2.20, \quad Q_{0}=158 ; \log \quad K=0.18, \quad K=0.66$. However, the procedure of elimination used in the preceding paragraph does not give weight to the discarded equations, and so does not give a best estimate of the parameters. It is useful mainly as a guide to a more judicious weighting.

Instead of following the exact procedure above, it is possible to write each of the equations in the set of the preceding section as written plus a correction 
term to be added to the constant term. A set of constant corrections is then sought whose magnitudes have the lowest upper bound, such that all the equations are consistent. Such a set can be formed by adding -0.095 to the constant in (12.3), 0.047 each to the identical equations (17.3) and (30.3), 0.11 to $(22.3), 0.094$ to $(29.3),-0.11$ to $(28.3)$, and 0.021 to the logarithms of $\bar{v}$ of (23). The method will be described more fully in the next section as it is applied in the two-body case.

Note that the equations (in nonlogarithmic form) have been altered by less than 13 percent. The simultaneous set (12.3), (30.3), and (33.3) then becomes

$$
\begin{aligned}
& 0.5 \log A+\log Q_{0}=2.96 \\
& -\log K+\frac{2}{3} \log A=1.39 \\
& \log K+\frac{1}{3} \log Q_{0}=0.62 .
\end{aligned}
$$

Solution of this set yields

$$
\begin{array}{ll}
\log A=2.04 & A=109 \cong 100 \mathrm{sec} \\
\log Q_{0}=1.95 & Q_{0}=89 \cong 100 \\
\log K=0.03 & K=0.97 \cong 1.0 .
\end{array}
$$

Because of the closeness of the above results to round numbers and because of their approximate nature, the values $A=100 \mathrm{sec}, Q_{0}=100$, and $K=1.0$ will be adopted as best estimates of the parameters. Together with the value $J=0$, these results imply that

$$
Q_{m o}=100 \times 10^{-M / 2.5}
$$

and is independent of velocity. The actual line density is $1.13 \times Q_{m_{0}} \mathrm{el} / \mathrm{m}$.

Not used in the simultaneous solution above were the results for $v_{60} / v_{35}$. For three-body attachment (10.3) gives this ratio as 1.82 , and (16) as 1.8 ; these results are consistent. The small adjustments required to obtain consistency of the whole set of three-body equations leads to the conclusion that the three-body attachment theory well explains all the data considered.

\section{Treatment of Two-Body Equations}

In this section the two-body equations will be examined for consistency. It will be shown that the two-body equations are considerably less consistent than are the three-body equations; the implication is that the two-body attachment theory does not as satisfactorily explain the experimental observations. The analysis will be based on the second procedure of the previous section. That is, an arbitrary correction term will be added to the constant term of each equation of the set, and the conditions on the constant will be sought leading to the lowest upperbound on the magnitude of the corrections.

Define correction terms to the constants in the equation by the following symbols: (12.2), a (that is, the constant becomes $3.98+a) ;(17.2), b$;) 20$)$, $c$; (30.2), d; (22.2), e; (23), let $\log \bar{v}=0.1+f ;(29.2)$, $g$; (28.2) and (31.2), let a combined constant be $1.42+m / 2$, and the difference is 0.03 . Then combining equations in the same sequence as was used for elimination in the three-body case, the following relations must hold in order that the whole set of equations be consistent:

$$
\begin{aligned}
& -a-\frac{6}{5} d=0.51-m / 2 \\
& -d-\frac{10}{3} f=0.29-g \\
& -a+\frac{6}{5} d+\frac{4}{5} f=1.60-e
\end{aligned}
$$

The constant $c$ appears only in (20) and so cannot be evaluated; let it equal zero. The above set of equations is to be solved by determining values of the constants $a$ through $m$ so that the greatest magnitude of any of the constants is as small as possible. By trial and error it has been found that the following approximate values satisfy the equations, and it does not appear that a set can be found with a smaller upper bound: the solution used is $a=-0.41, d=0.1, e=0.39, m=0.42, g=0.39$, $f=0$. These values are four times as large as the greatest correction needed in the three-body case. In addition, $v_{60} / v_{35}=2.42$ from (10.2) may be compared with $v_{60} / v_{35}=1.8$ from (16). The check obtained in the three-body case (1.82 versus 1.8$)$ is not obtained in the two-body case.

Although the greater inconsistency of the two-body equations makes the results of the two-body analysis appear to be unrealistic, solution of the adjusted set can be effected using the altered values of (12.2), (30.2), and (20) with $\log \bar{v}=0.1+f$ from (23). The set becomes, with $c$ zero,

$$
\begin{aligned}
\log A+\log Q_{0} & =3.78 \\
-\log K+\frac{4}{3} \log A & =1.76 \\
\log K+\frac{1}{3} \log Q_{0} & =0.55
\end{aligned}
$$

The solution is $\log Q_{0}=2.73, Q_{0}=540 ; \log A=1.05$, $\mathrm{A}=11.2 ; \log K=-0.36, K=.044$. Note that $A$, the attachment time constant at the reference height of $95 \mathrm{~km}$, is not a comparable quantity for two- and three-body attachment, since the height variation of the time constant is different.

\section{Discussion}

Greenhow and Hall [1961] concluded that threebody rather than two-body attachment is the probable method of meteoric charge removal because of $(a)$ the rapidity with which the luminosity of visual trains decay at lower heights, and $(b)$ the infrequent occurrence of long-enduring radio echoes at heights much below $90 \mathrm{~km}$. However, they 
considered the radio data to fit the theory equally well for either attachment law. Since they did not publish a detailed mathematical theory, their calculations cannot be checked or extended. Comparison of the normalizing factors given by Greenhow and Hall for the plotting of duration versus visual magnitude shows that their theoretical relations could not have been consistent with those in the present work. Thus in our notation, Greenhow and Hall in their figure 3 normalize three-body duration by a factor proportional to $A V^{3} \lambda^{3 / 5}$, while the present theory requires a factor of $A^{4 / 5} V^{2} \lambda^{2 / 5}$; their magnitude is normalized by a constant plus log $\left(\lambda^{3} / V^{11.25}\right)$, while the present theory gives $\log \left[\lambda^{2.5} /\right.$ $\left.\left(A^{1.25} V^{12.5}\right)\right]$. Note especially the absence of the attachment time constant in Greenhow and Hall's expression for the normalization of magnitude. Similar differences exist in the two-body normalization.

Because of its analytic basis, it has been possible to use the theory of part I to compare the various experimental results with each other and thus to arrive at values not only of the attachment time constant $A$, but also for the constants $J, K$, and $Q_{0}$ appearing in the full theory. Moreover, it has been found that the three-body process is strongly favored in comparison with the two-body process on the basis of the consistency of experiments of different types. Such a comparison was not made by Greenhow and Hall.

The most probable value for $J$, the exponent of the velocity dependence of the ratio of line density to luminosity was found to be zero. Together with the value of $Q_{0}$ from $\left(35.3^{*}\right)$ the results imply that the maximum initial line density on a trail in electrons $/ \mathrm{m}$ is quite well represented by $1.13 \times 10^{14}$ times the normalized density

$$
Q_{m o}=100 \times 10^{-M / 2.5}
$$

where $M$ is the visual magnitude. Using the weighted two-body fit, the value of the constant would be 540 ; the three-body theory is preferred.

The value of $K=1.0$ was adopted from the three body reduction ( $K=0.44$ was found from the twobody theory). With $J=0, K=1.0$ placed in the defining equation, (11) of part I yields

$$
\exp \frac{95-h_{m o}}{H}=\frac{Q_{m o}^{1 / 3}}{V^{10 / 3}}
$$

where $V$ is the meteoric velocity in $\mathrm{km} / \mathrm{sec}, Q_{m_{o}}$ is $0.885 \times 10^{-14}$ times the maximum initial line density in electrons $/ \mathrm{m}, M$ is the atmospheric scale height in $\mathrm{km}$, and $h_{m_{o}}$ is the height of maximum initial line density in $\mathrm{km}$.

In the theory of part I echo duration was described by the normalized expression $z$, and line density by the normalized line-density expression $x$. Using the three-body values $A=100$ sec, $K=1.0, D=4 \mathrm{~m}^{2} / \mathrm{s}$, and $J=0,(1.3)$ and (2.3) become

$$
z=\frac{0.281}{\lambda^{2 / 3} v^{10 / 3}} t_{H}
$$

and

$$
x=\frac{\lambda}{148 v^{5}} Q_{m o}=\frac{\lambda 10^{-M / 2.5}}{148 v^{5}} .
$$

When $\lambda=10 \mathrm{~m}$, the expression for $z$ simplifies to $z=t_{H} /\left(16.5 v^{10 / 3}\right)$.

The transition between attachment-free and attachment-controlled duration centers about $z=1 / 2$ and $x=2$. Thus the transition durations and line densities are approximately $t_{H}=1.8 \lambda^{2 / 3} v^{10 / 3}$, and $Q_{m o}=300 v^{5} / \lambda$. If $\lambda=10 \mathrm{~m}$, the transition centers about $t_{M}=8.3 v^{10 / 3} \mathrm{sec}, Q_{m_{0}}=30 v^{5}$. The transition extends over about a ten-to-one line-density range.

Although the theories have been described in terms of "two-body" or "three-body" attachment, the mathematics of the analysis is based merely on the assumption that the initial line density decreases exponentially with time, and that the time constant of the decay varies exponentially with height either as $\exp (h / H)$, or else as $\exp (2 h / H)$. The latter variation has been found more consistent with experiments and has led to the factor exp $[-t$ $\left.\left(100 e^{2(h-95) / H}\right)\right]$ by which the initial line density at height $h \mathrm{~km}$ should be multiplied. The line density versus time, magnitude, and height can be found using $Q_{m_{o}}$ from $\left(40.3^{*}\right), h_{m o}=95 \mathrm{~km}, A=100 \mathrm{sec}$, and (7) of part I with $m=2$.

If the rate of loss of line density is attributed to a simple three-body attachment process, the rate coefficient $k$ is defined by $d N / d t=-k n^{2} N$ where $n$ is the atmospheric number density. Thus $A=1 / \mathrm{kn}^{2}$, and using $A=100 \mathrm{sec}, n=2.1 \times 10^{12} \mathrm{~cm}^{-3}$ from the 1959 ARDC model atmosphere, $k=2.2 \times 10^{-27} \mathrm{~cm}^{6} /$ sec. Assuming two-body attachment, $d N / d t=$ $-k n N$, and with $A=11.2, k=4.2 \times 10^{-14} \mathrm{~cm}^{3} / \mathrm{sec}$. Neither coefficient is beyond the range of possible values for attachment processes; however, the two-body rate is perhaps a little closer to consistency with published values. The value $A=100 \mathrm{sec}$ may be compared with the value of $70 \mathrm{sec}$ adopted by Greenhow and Hall, and the value of $11.2 \mathrm{sec}$ for the two-body process compared with their adopted value of 40 sec. The values of the coefficients are quite sensitive to errors in the experimental results.

If the present attachment theory of meteor durations is accepted, eddy diffusion must be discounted as a factor influencing the duration of meteor echoes lasting as long as 100 sec. Greenhow [1959] has published an interpolation between the rates of expansion of radio trails and visual trains that implies that $100 \mathrm{sec}$ after trail formation the effective diffusion coefficient is more than ten times as large as the ambipolar coefficient. Such a result appears incompatible with the attachment theory, either as given here or by Greenhow. Reasons exist for believing a different interpretation may be given to the discrepancy between the radio and visual train sizes. 


\section{Conclusion}

By developing the theory of meteor-echo duration including attachment in a general form, it has been possible to study the mutual consistency of the various types of experimental results available relating meteor magnitude, height, echo duration, and the wavelength of the sounding radio waves. It has been found that the experimental results are in considerably better agreement with a three-body than with a two-body attachment theory. Values have been deduced for the attachment rate, the initial line density of the trail versus visual magnitude, for a constant relating height of maximum line density to the velocity and intensity of ionization, and for the velocity dependence of the ratio of ionization to luminosity. It is believed that the method used for comparing the results of experiments of different types leads to more consistent values for the constants of the theory of meteor durations than have previously been available. In combination with the theoretical results of part I, use of the constants deduced in the present paper makes possible the theoretical prediction and description of radio echo heights and durations.

\section{References}

Davis, J., J. S. Greenhow, and J. E. Hall (1959a), Combined photographic and radio echo observations of meteors, Proc. Roy. Soc. 253, No. 1272, 121-129.

Davis, J., J. S. Greenhow, and J. E. Hall (1959b), The effect of attachment on radio echo observation of meteors, Proc. Roy. Soc. 253, No. 1272, 130-139.

Greenhow, J. S. (1952), Characteristics of radio echoes from meteor trails, Proc. Phys. Soc. 65, 196-181.

Greenhow, J. S. (1959), Eddy diffusion and its effect on meteor trails, J. Geophys. Res. 64, 2208-2209.

Greenhow, J. S., and J. E. Hall (1961), Attachment processes in meteor trails, J. Atmospheric Terrest. Phys. 21, 261-271.

Manning, L. A. (1964), The theoretical heights and durations of echoes from large meterors, Radio Sci. J. Res. NBS/USNCURSI 68D, No. 10, 1067-1078.

McKinley, D. W. R. (1953), Meteor echo duration and radio wave length, Can. J. Phys. 31, 1121-1135.

McKinley, D. W. R. (1961), Meteor Science and Engineering (McGraw-Hill Book Co., Inc., New York, N.Y.).

Millman, P. M., and D. W. R. McKinley (1956), Meteor echo durations and visual magnitudes, Can. J. Phys. 34, 50-61.

(Paper 68D10-408) 